\title{
Analysis of the Contribution of Large Scale Motions to the Skin Friction of a Zero-Pressure-Gradient Turbulent Boundary Layer Using the Renard-Deck Decomposition
}

\author{
B. Sun ${ }^{1 *}$, M. Shehzad ${ }^{1}$, D. Jovic ${ }^{1}$, C. Cuvier ${ }^{2}$, C. Willert ${ }^{3}$, Y. Ostovan ${ }^{2}$, \\ JM. Foucaut ${ }^{2}$, C. Atkinson ${ }^{1}$, J. Soria ${ }^{1}$ \\ ${ }^{1}$ Laboratory for Turbulence Research in Aerospace \& Combustion (LTRAC), Department of Mechanical \\ and Aerospace Engineering, Monash University, Victoria 3800, Australia \\ ${ }^{2}$ Université Lille Nord de France, LMFL - Laboratoire de Mécanique des Fluides de Lille, FRE CNRS \\ 2017, Centrale Lille, ENSAM, ONERA, Villeneuve d'Ascq, France \\ ${ }^{3}$ Institute of Propulsion Technology, German Aerospace Center (DLR), Cologne, Germany \\ *bihai.sun@monash.edu
}

\begin{abstract}
Coherent flow structures in turbulent boundary layers have been an active field of research for many decades, as they might be the key to reveal the mechanics of turbulence production and transport in turbulent shear flows. Renard and Deck (2016) proposed a theoretical decomposition for the mean skin-friction coefficient based on the mean kinetic energy budget in the streamwise direction. This decomposition, referred to as the Renard-Deck (RD) decomposition, decomposes the mean skin friction generation into three physical mechanisms in an absolute reference frame, namely, direct viscous dissipation, turbulent kinetic energy production, and spatial growth. In this study, the large scale motions (LSMs) are extracted using a proper orthogonal decomposition (POD) of the velocity field based on high-spatial-resolution two-dimensional two-component particle image velocimetry (HSR 2C-2D PIV) of a zero-pressure-gradient turbulent boundary layer (ZPG-TBL), and their effect on the skin friction via RD decomposition.

The high Reynolds number turbulent boundary layer experiment was performed at the Laboratoire de Mécanique des Fluides de Lille (LMFL) in the LMFL High Reynolds Number Boundary Layer Wind Tunnel. The measurement took place in the streamwise - wall-normal plane along the centreline of the wind tunnel, where the ZPG-TBL at the measurement location has a free-stream velocity of $9 \mathrm{~m} / \mathrm{s}$, a Reynolds number based on the momentum thickness of $R e_{\theta}=8,120$, a boundary layer thickness of $\delta \approx 103 \mathrm{~mm}$ and a viscous length of $l^{+} \approx 40 \mu \mathrm{m}$. The PIV images were captured with a 47MP Imperx Tiger T8810 camera which has a $57 \mathrm{~mm}$-diagonal array of size $8,864 \times 5,288$ pixels. This results in a field of view of $255 \mathrm{~mm} \times 152 \mathrm{~mm}$, or $2.46 \delta \times 1.45 \delta$ in terms of the boundary layer thickness, with a spatial resolution of 21.7 wall units in the streamwise direction and 5.42 wall units in the wall-normal direction. A more detailed description of the experiment, as well as an analysis on the method to correct for the lens distortion introduced by the large sensor size, can be found in Sun et al. (2021).

The LSMs present in the ZPG-TBL are extracted from the velocity field using POD by the snapshot method (Sirovich, 1987). Due to the limited field of view of the acquired data, the LSMs in the present study are defined as the flow structures that have a wall-normal extent of approximately the full boundary layer, which are identified by the most energetic POD mode as shown in figure 1a. This mode accounts for $16 \%$ of the turbulent kinetic energy in the flow. Velocity field snapshots containing stronger LSMs than others are identified using the time coefficient of the most energetic POD mode, $\phi_{1}$, whose distribution is presented in figure $1 \mathrm{~b}$. The distribution of $\phi_{1}$ is Gaussian-like, with its absolute value representing the strength of the LSM present in each snapshot. Therefore, the following criterion is used to determine the snapshots containing strong LSMs,
\end{abstract}

$$
\left|\phi_{1, n}\right| \geq K \sigma_{\phi_{1}},
$$


where $\phi_{1, n}$ represents the time coefficient of the most energetic POD mode for snapshot $n$, and $\sigma_{\phi_{1}}$ represents the standard deviation of the time coefficient for all snapshots. A higher $\mathrm{K}$ value represents a tighter selection criterion.

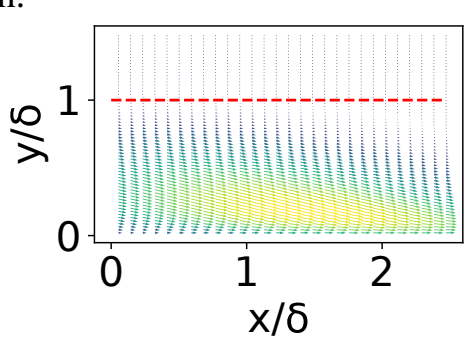

(a)

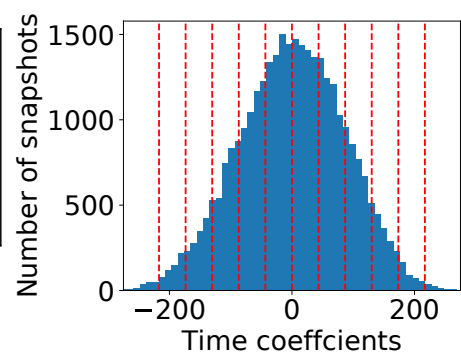

(b)

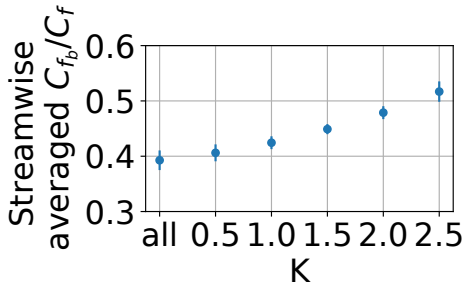

(c)

Figure 1: (a) A vector plot of the fluctuating velocity of the most energetic POD mode. The colours of the vectors represent the velocity fluctuation magnitude. The red dashed line represents the boundary layer thickness. (b) Distribution of the time coefficient of the most energetic POD mode, $\phi_{1}$. The red dashed line represents $\pm 2.5 \sigma, \pm 2 \sigma, \pm 1.5 \sigma, \pm \sigma, \pm 0.5 \sigma$ and the mean value of $\phi_{1}$. (c) Streamwise averaged $C_{f_{b}} / C_{f}$ for different $\mathrm{K}$ values. The error bars in $C_{f_{b}} / C_{f}$ represents its standard deviation along the streamwise direction.

Renard and Deck (2016) decomposition (RD decomposition) of the skin friction coefficient based on the mean kinetic energy budget of the fluid motion in an absolute frame of reference, which is given by

$$
C_{f}=\underbrace{\frac{2}{U_{e}^{3}} \int_{0}^{\infty} \mu\left(\frac{\partial U}{\partial y}\right)^{2} d y}_{C_{f_{a}}}+\underbrace{\frac{2}{U_{e}^{3}} \int_{0}^{\infty}-\left\langle u^{\prime} v^{\prime}\right\rangle \frac{\partial U}{\partial y} d y}_{C_{f_{b}}}+\underbrace{\frac{2}{U_{e}^{3}} \int_{0}^{\infty}\left(U-U_{e}\right) \frac{\partial}{\partial y}\left(\frac{\tau}{\rho}\right) d y}_{C_{f_{c}}},
$$

where $\tau=\mu(\partial U / \partial y)-\left\langle u^{\prime} v^{\prime}\right\rangle, U$ denotes the mean streamwise velocity, $U_{e}$ denotes the free stream velocity, $\left\langle u^{\prime} v^{\prime}\right\rangle$ denotes the Reynolds shear stress, $\mu$ denotes the dynamic viscosity and $\rho$ denotes the density. The $\mathrm{RD}$ decomposition decomposes the skin friction into physically interpretable terms that are local at each streamwise position. The first term, $C_{f_{a}}$ represents the viscous dissipation of the mean streamwise kinetic energy, and it depends only on the mean values of the fluid field therefore has no direct contribution from the LSMs. The second term, $C_{f_{b}}$, represents the production of the turbulent kinetic energy extracted from the mean streamwise kinetic energy and is the term of relevance in the analysis of the contribution of LSMs. The last term, $C_{f_{c}}$, accounts for the growth of the boundary layer effect, as the growth of the boundary layer is slow for a ZPG-TBL, this term is negligible. A conditional statistics analysis is performed by calculating the $C_{f_{b}}$ term using the snapshots containing strong LSMs as identified by equation 1 . The resulting streamwise averaged value of $C_{f_{b}} / C_{f}$ for different threshold value $\mathrm{K}$ is presented in figure $1 \mathrm{c}$. This results show that LSM dominated snapshots results in an increase of $C_{f_{b}}$, indicating that the LSMs are a significant contributor to the turbulent production term in the RD decomposition of the skin friction.

\section{Acknowledgements}

This research was supported by the Australian Government through the Australian Research Council's Discovery Projects funding scheme. The research was also benefited from computational resources provided by the Pawsey Supercomputing Centre and through NCMAS, supported by the Australian Government. The computational facilities supporting this project included the NCI Facility, the partner share of the NCI facility provided by Monash University through an ARC LIEF grant and the Multi-modal Australian ScienceS Imaging and Visualisation Environment (MASSIVE). Daniel Jovic and Bihai Sun are supported by a Research Training Program (RTP) scholarship provided by the Australian government.

\section{References}

Renard N and Deck S (2016) A theoretical decomposition of mean skin friction generation into physical phenomena across the boundary layer. Journal of Fluid Mechanics 790:339-367

Sirovich L (1987) Turbulence and the dynamics of coherent structures. I. Coherent structures. Quarterly of Applied Mathematics 45:561-571

Sun B, Shehzad M, Jovic D, Cuvier C, Willert C, Ostovan Y, Foucaut JM, Atkinson C, and Soria J (2021) Distortion correction of two-component - two-dimensional PIV using a large imaging sensor with application to measurements of a turbulent boundary layer flow at $R e_{\tau}=2,386$. Experiment in Fluids 